\title{
Evolutionary Dynamics on Graphs: Efficient Method for Weak Selection
}

\section{Citation}

Fu, Feng, Long Wang, Martin A. Nowak, and Christoph Hauert. 2009. Evolutionary dynamics on graphs: Efficient method for weak selection. Physical Review E 79(4): 046707.

\section{Published Version}

doi:10.1103/PhysRevE.79.046707

\section{Permanent link}

http://nrs.harvard.edu/urn-3:HUL.InstRepos:4065630

\section{Terms of Use}

This article was downloaded from Harvard University's DASH repository, and is made available under the terms and conditions applicable to Other Posted Material, as set forth at http:// nrs.harvard.edu/urn-3:HUL.InstRepos:dash.current.terms-of-use\#LAA

\section{Share Your Story}

The Harvard community has made this article openly available.

Please share how this access benefits you. Submit a story.

\section{Accessibility}




\title{
Evolutionary dynamics on graphs: Efficient method for weak selection
}

\author{
Feng Fu, ${ }^{1,2}$ Long Wang, ${ }^{2}$ Martin A. Nowak, ${ }^{1,3}$ and Christoph Hauert ${ }^{1,4, *}$ \\ ${ }^{1}$ Program for Evolutionary Dynamics, Harvard University, Cambridge, Massachusetts 02138, USA \\ ${ }^{2}$ Center for Systems and Control, State Key Laboratory for Turbulence and Complex Systems, \\ College of Engineering, Peking University, Beijing 100871, China \\ ${ }^{3}$ Department of Organismic and Evolutionary Biology and Department of Mathematics, \\ Harvard University, Cambridge, Massachusetts 02138, USA \\ ${ }^{4}$ Department of Mathematics, University of British Columbia, Vancouver, British Columbia, Canada V6T $1 Z 2$ \\ (Received 17 September 2008; revised manuscript received 2 March 2009; published 13 April 2009)
}

\begin{abstract}
Investigating the evolutionary dynamics of game theoretical interactions in populations where individuals are arranged on a graph can be challenging in terms of computation time. Here, we propose an efficient method to study any type of game on arbitrary graph structures for weak selection. In this limit, evolutionary game dynamics represents a first-order correction to neutral evolution. Spatial correlations can be empirically determined under neutral evolution and provide the basis for formulating the game dynamics as a discrete Markov process by incorporating a detailed description of the microscopic dynamics based on the neutral correlations. This framework is then applied to one of the most intriguing questions in evolutionary biology: the evolution of cooperation. We demonstrate that the degree heterogeneity of a graph impedes cooperation and that the success of tit for tat depends not only on the number of rounds but also on the degree of the graph. Moreover, considering the mutation-selection equilibrium shows that the symmetry of the stationary distribution of states under weak selection is skewed in favor of defectors for larger selection strengths. In particular, degree heterogeneity - a prominent feature of scale-free networks - generally results in a more pronounced increase in the critical benefit-to-cost ratio required for evolution to favor cooperation as compared to regular graphs. This conclusion is corroborated by an analysis of the effects of population structures on the fixation probabilities of strategies in general $2 \times 2$ games for different types of graphs. Computer simulations confirm the predictive power of our method and illustrate the improved accuracy as compared to previous studies.
\end{abstract}

DOI: 10.1103/PhysRevE.79.046707

PACS number(s): 05.10.-a, 87.23.Kg, 89.75.Hc, 02.50.Le

\section{INTRODUCTION}

Evolutionary game dynamics on graphs [1-7] has attracted growing interests in different fields [8-22] (for a recent review see [23]) as a significant extension of traditional evolutionary game theory focusing on well-mixed populations [24-27]. In games on graphs, individuals are located on the vertices of a graph. The edges determine interactions and competition among individuals. Specifically, consider a population of two strategic types of individuals, $A$ and $B$, on an arbitrary graph. Each individual engages in pairwise interactions with all its neighbors and accumulates a total payoff, $\Pi$. The interactions are characterized by the $2 \times 2$ payoff matrix $M_{X Y}(X, Y=\{A, B\})$, which specifies the payoffs to the row player

$$
{ }_{B} \begin{array}{cc}
A & B \\
B & \left(\begin{array}{ll}
M_{A A} & M_{A B} \\
M_{B A} & M_{B B}
\end{array}\right) .
\end{array}
$$

The evolutionary success of an individual is determined by its fitness, which may be given by a convex combination of a baseline fitness normalized to 1 and the payoff $\Pi, 1-w$ $+w \Pi$, where $w \in[0,1]$ measures the intensity of selection, i.e., the relative contribution of the game to fitness. It should

\footnotetext{
*christoph.hauert@math.ubc.ca
}

be noted that an individual fitness never becomes negative for the small values of $w$ used in this paper (i.e., from 0.0001 to 0.05$)$. For weak selection $(w \ll 1)$, the payoff obtained from game interactions makes a small contribution to the overall fitness of an individual. This situation can be justified in two ways: (i) the results derived from weak selection often remain as valid approximations for larger selection strength $[5,6]$; (ii) the weak selection limit has a long tradition in theoretical biology (i.e., closeness in phenotype space) when working with population genetics [28] and kin selection theory [29]. Indeed, inclusive fitness analysis exclusively relies on the assumption of weak selection (see Ref. [7] and references therein). Individuals with a high fitness have a high propensity to proliferate and transmit their strategies either through genetic inheritance or through cultural imitation. The evolutionary outcome crucially depends on the specific updating mechanism used [5]. In this paper, we adopt death-birth updating; in each time step the neighbors of a randomly selected focal individual compete to pass their strategy to the focal individual. One neighbor succeeds with a probability proportional to its fitness. This represents a spatial analog of the Moran process [30] with frequencydependent fitness. Thus, the population size, $N$, remains constant during the evolutionary process.

Over the years, a number of different and biologically relevant updating rules have been proposed and investigated in the context of spatial games. Most prominently this includes pairwise comparison processes [26] as well as birth-death and death-birth processes inspired by the Moran 
process [30]. It turns out that in the limit of weak selection, pairwise comparison processes are equivalent to the birthdeath process [31] — but for this process it has already been shown in [5] that it never favors cooperation in the prisoner dilemma on graphs. For this reason the following analysis focuses on the death-birth process, which is able to sustain cooperation even in the limit of weak selection. Nevertheless, it is certainly worth exploring further updating mechanisms for strong selection. In this situation, however, other complicated issues arise. For example, fixation probabilities may no longer be the best way to characterize the dynamics of the population because strategies may coexist for very long times. Even more importantly, the accounting of the payoffs matters on heterogeneous graphs. That is, the results would be qualitatively different if the fitness of each individual is based on the accumulated payoffs from interactions with all neighbors or if it is, e.g., based on a single interaction with a randomly chosen neighbor. Consequently, for practical and illustrative purpose, we restrict our focus on the death-birth updating rule.

In general, the wealth of conceivable population structures, together with the exceedingly large number of possible configurations, makes analytical investigations almost intractable. In most instances, simulations are used instead and considerable attention has been paid to the case of strong selection $[9,10]$. It turns out that graph topologies play a decisive role in the evolutionary process $[4,18,19]$. Fortunately, the limit of weak selection leads to a natural separation of timescales regarding local and global dynamics; that is, the local frequency of players equilibrates much faster than the global density of players. Therefore, weak selection allows for analytic estimations based on pair approximation $[5,32]$ or inclusive fitness [7] analysis. We should note that the former method is exact for infinitely large regular graphs without loops, e.g., trees or Bethe lattices, while the latter is valid for payoff matrices satisfying "equal gains from switching" (i.e., $M_{A A}+M_{B B}=M_{A B}+M_{B A}$ [33]) on regular graphs satisfying particular homogeneity properties. As a result, neither of these two methods is capable of dealing with arbitrary degree-heterogeneous graphs. Furthermore, determining the evolutionary outcome empirically requires extremely time-consuming simulations.

Perturbation approaches have proven to be efficient and effective to substantially reduce the complexity of computation. For instance, perturbation analysis predicts the equilibrium behavior of systems of interacting players in spatial games and particles in lattice-gas models in the limit of zero noise (i.e., low temperature in statistics physics). These predictions can be obtained by perturbing deterministic evolution by suitable stochastic terms and performing a lowtemperature (low-noise) expansion [34,35]. In contrast, the limit of weak selection corresponds to the high-temperature case. In analogy, the neutral (random) evolutionary dynamics of the population can be perturbed to account for game theoretical interactions among individuals. In this sense the weak selection limit represents a perturbation analysis, which is applicable to any type of game and also applies to degree-heterogeneous graphs.
For weak selection, population configurations are nearly independent of the game but remain affected by the underlying graph topology. Therefore, local configurations can be approximated by neutral evolution on graphs. For a restricted set of graphs, this allows one to estimate correlations between $A$ and $B$ types in the case of pair approximation [5] and the relatedness among neighbors for inclusive fitness analysis [7]. Here we determine the evolutionary dynamics for arbitrary games on any graph by tracking the microscopic dynamics along the interface between $A$ and $B$ types based on empirically determined correlations between the two types under neutral evolution. Hence, under weak selection, the game dynamics represents a first-order correction to the dynamics under neutral evolution.

\section{METHODS}

In neutral populations we determine through simulations the number of $A A, A B$, and $B B$ links as a function of the number $i$ of $A$ types, denoted by $N_{A A}(i), N_{A B}(i)$, and $N_{B B}(i)$, respectively. The average degree of $A$ and $B$ individuals is then given by $k_{A}(i)=\left[2 N_{A A}(i)+N_{A B}(i)\right] / i=n_{A}^{A}(i)+n_{A}^{B}(i)$ and $k_{B}(i)=\left[2 N_{B B}(i)+N_{A B}(i)\right] /(N-i)=n_{B}^{A}(i)+n_{B}^{B}(i), \quad$ respectively, where $n_{X}^{Y}(i)$ denotes the average number of $X Y$ links of an $X$ individual. Based on these quantities, we can approximate an individual payoff for the game dynamics, if present. Since we consider death-birth updating, we only need to determine the payoffs of the neighbors that compete to repopulate the vacant focal site. In analogy to the moment closure in pair approximation, we approximate the average number of $X$ neighbors around a $Y$ individual as $1+\left[\left(k_{Y}-1\right) / k_{Y}\right] n_{Y}^{X}$, given that the $Y$ individual has at least one $X$ neighbor. Therefore, the average payoff of a $Y$ player in the neighborhood of the focal individual, $X$, can be approximated as follows:

$$
\begin{aligned}
\Pi_{X}^{Y}(i)= & {\left[\delta_{X, A}+\frac{k_{Y}(i)-1}{k_{Y}(i)} n_{Y}^{A}(i)\right] M_{Y A} } \\
& +\left[\delta_{X, B}+\frac{k_{Y}(i)-1}{k_{Y}(i)} n_{Y}^{B}(i)\right] M_{Y B},
\end{aligned}
$$

where $\delta_{X, Y}$ is equal to 1 if $X=Y$, and 0 otherwise. Hence, the corresponding fitness reads $F_{X}^{Y}(i)=1-w+w \Pi_{X}^{Y}(i)$.

Evolutionary changes can only occur along the interface of $A$ and $B$ types. The microscopic dynamics can thus be described as follows. If the number of $A$ 's increases from $i$ to $i+1$, the average number of $A$ 's and $B$ 's in the neighborhood of a vacated $B$ site along the boundary, $k_{B}^{A}(i)$ and $k_{B}^{B}(i)$, is determined by the changes in the number of $A A$ and $B B$ links: $k_{B}^{A}(i)=N_{A A}(i+1)-N_{A A}(i)$ and $k_{B}^{B}(i)=N_{B B}(i)-N_{B B}(i+1)$ with $i=1, \ldots, N-2$. With only a single $B(i=N-1)$, we have $k_{B}^{A}(N-1)=N_{A B}(N-1)$ and $k_{B}^{B}(N-1)=0$. In analogy, if the number of $A$ types decreases from $i+1$ to $i$, the average number of $A$ 's and $B$ 's in the neighborhood of a vacated $A$ site along the boundary is $k_{A}^{A}(i+1)=k_{B}^{A}(i)$ and $k_{A}^{B}(i+1)$ $=k_{B}^{B}(i)$ for $i=1, \ldots, N-2$ as well as $k_{A}^{A}(1)=0$ and $k_{A}^{B}(1)$ $=N_{A B}(1)$ for a single $A(i=1)$. Consequently, the number of $A$ 's and $B$ 's along the interface is given by $n_{A}(i)$ $=N_{A B}(i) / k_{A}^{B}(i)$ and $n_{B}(i)=N_{A B}(i) / k_{B}^{A}(i)$. This results in a 


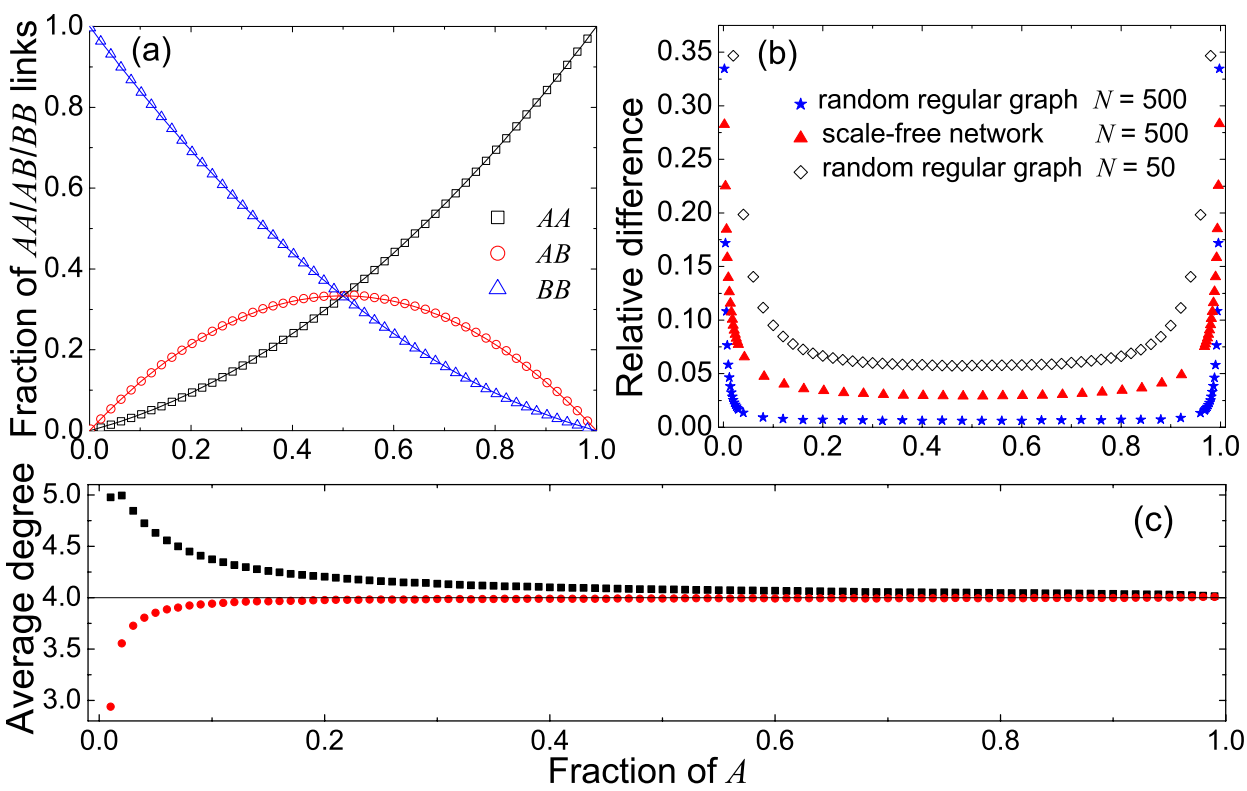

FIG. 1. (Color online) Neutral evolution on graphs. (a) The equilibrium fraction of $A A(\square), A B(\bigcirc)$, and $B B(\triangle)$ links as a function of the fraction of $A$ individuals on random regular graphs. The solid lines correspond to pair-approximation results. (b) The relative difference of the empirically determined fraction of $A B$ links to that of pair approximation. (c) Invasion process on scale-free networks starting from a single randomly placed $A$. Average degree of $A$ when the number of $A$ increases ( $\boldsymbol{\square})$ or decreases $(\boldsymbol{)})$. [Population size (a) $N=500$, (b) 500 $(\star, \mathbf{\Delta}), 50(\diamond)$, and (c) 100 and average degree $k=4$. All data points are averaged over $10^{7}$ runs.]

detailed description of the environment along the $A B$ interface, which in turn determines the evolutionary change.

The evolutionary dynamics on graphs represents a discrete Markov process on the interval $[0, N]$ with states 0 and $N$ being absorbing. At each time step, the number of $A$ individuals, $i$, can increase by one, decrease by one, or stay the same. Therefore, the transition matrix of this process is given by

$$
\begin{aligned}
& T_{A}^{+}(i)=\frac{n_{B}(i)}{n_{A}(i)+n_{B}(i)} \frac{k_{B}^{A}(i) F_{B}^{A}(i)}{k_{B}^{A}(i) F_{B}^{A}(i)+k_{B}^{B}(i) F_{B}^{B}(i)}, \\
& T_{A}^{-}(i)=\frac{n_{A}(i)}{n_{A}(i)+n_{B}(i)} \frac{k_{A}^{B}(i) F_{A}^{B}(i)}{k_{A}^{A}(i) F_{A}^{A}(i)+k_{A}^{B}(i) F_{A}^{B}(i)},
\end{aligned}
$$

where $T_{A}^{+}, T_{A}^{-}$indicates the probability that $i$ increases $(i \rightarrow i$ $+1)$ or decreases $(i \rightarrow i-1)$, respectively. With probability $T_{A}^{0}(i)=1-T_{A}^{+}(i)-T_{A}^{-}(i), i$ remains constant. All other entries of the transition matrix are 0 . We denote by $x_{i}$ the fixation probability of $A$ when starting from $i A$ 's. We have

$$
x_{i}=T_{A}^{+}(i) x_{i+1}+T_{A}^{0}(i) x_{i}+T_{A}^{-}(i) x_{i-1},
$$

with boundary conditions $x_{0}=0$ and $x_{N}=1$.

In order to solve above recursive equation, rewriting Eq. (4) we have

$$
x_{i+1}-x_{i}=\frac{T_{A}(i)}{T_{A}^{+}(i)}\left(x_{i}-x_{i-1}\right)=\prod_{j=1}^{i} \frac{T_{A}(j)}{T_{A}^{+}(j)}\left(x_{1}-x_{0}\right) .
$$

Summing Eq. (5) from $i=1$ to $i=N-1$ we have

$$
x_{N}-x_{1}=\sum_{i=1}^{N-1} \prod_{j=1}^{i} \frac{T_{A}^{-}(j)}{T_{A}^{+}(j)}\left(x_{1}-x_{0}\right) \text {. }
$$

Let $\rho_{A}\left(\rho_{B}\right)$ denote the probability that a single randomly placed $A(B)$ individual in an otherwise homogeneous population of $B(A)$ types reaches fixation in the absence of mutation. We thus have $\rho_{A}=x_{1}$ and $\rho_{B}=1-x_{N-1}$. According to Eqs. (5) and (6) we obtain $[24,36]$

$$
\rho_{A}=\frac{1}{1+\sum_{i=1}^{N-1} \prod_{j=1}^{i} T_{A}(j) / T_{A}^{+}(j)}
$$

and

$$
\frac{\rho_{A}}{\rho_{B}}=\prod_{i=1}^{N-1} \frac{T_{A}^{+}(i)}{T_{A}^{-}(i)} .
$$

If $\rho_{A}>1 / N, A$ has a higher fixation probability than a neutral mutant. If, in addition, $\rho_{A}>\rho_{B}$ holds, $A$ has a selective advantage over $B$ such that in the long run and for rare mutations, the population is more likely to consist of $A$ individuals only.

\section{RESULTS AND DISCUSSION}

Specifically, consider neutral evolution on random regular graphs [37] as well as on scale-free networks [38] (Fig. 1). The deviation between simulation data and pair approximation is evidently no longer negligible for small populations or heterogeneous numbers of neighbors [Figs. 1(a) and 1(b)]. The invasion dynamics on regular random graphs is independent of the location where the mutant occurs, and thus can be seen as an unbiased random walk, namely, the probability of increasing or decreasing the number of mutants is the same. The invasion process of a single randomly placed neutral 


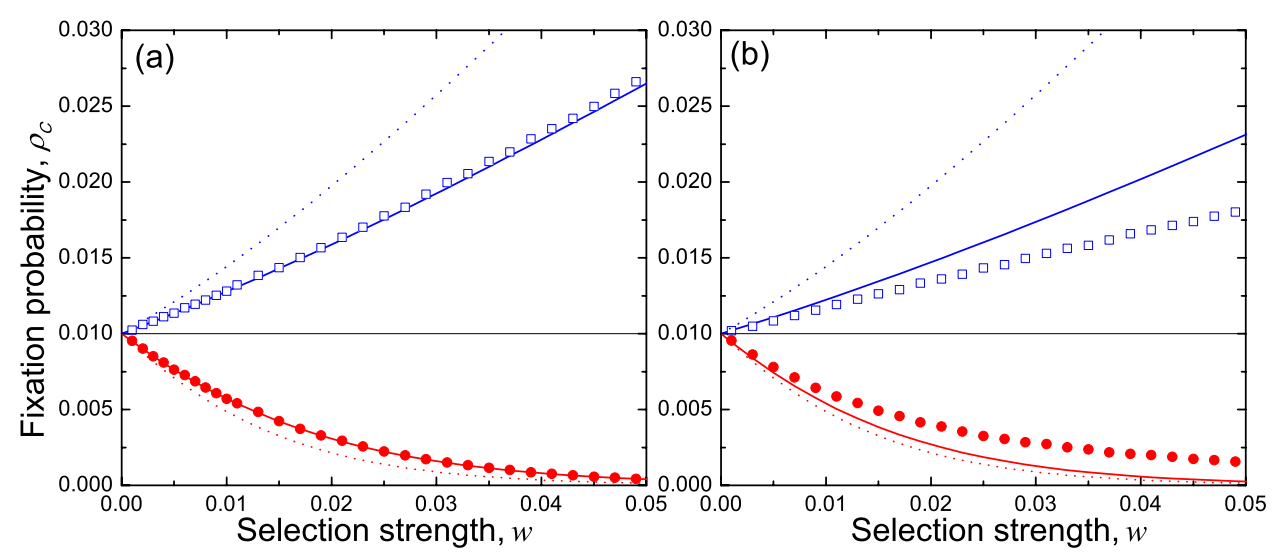

FIG. 2. (Color online) Fixation probability of a single randomly placed cooperator, $\rho_{C}$, in the prisoner dilemma on (a) random regular graphs and (b) scale-free networks. The fixation probability from simulations with benefit-to-cost ratio, $b / c=10(\square)$ and $2(\bullet)$, respectively, is determined by the fraction of runs where cooperators reached fixation out of $10^{7}$ runs. The upper (lower) solid line corresponds to the fixation probability obtained from our approach for the case of $b / c=10(b / c=2)$. The upper (lower) dotted line corresponds to the pair-approximation results for $b / c=10(b / c=2)$. The middle horizontal solid line indicates the fixation probability $1 / N$ under neutral evolution. $(N=100$ and $k=4$.)

mutant $A$ in a $B$ population becomes particularly interesting on scale-free networks [Fig. 1(c)]. In the initial phase, the fate of $A$ 's hinges on the odds to occupy large-degree nodes (hubs). The colony of $A$ is more likely to expand if $A$ individuals successfully control the hubs. Conversely, if $A$ individuals are constrained to small-degree nodes they are more likely to disappear. Thus, a rare mutant $A$ is prone to extinction unless the mutation occurs in a hub or the mutant successfully colonizes a hub. However, a randomly placed mu$\operatorname{tant} A$ is most likely to appear on small-degree nodes, since most nodes have few neighbors in scale-free networks. In this sense, degree heterogeneity opposes the initial spread of mutants and thus creates a high invasion barrier. This result has been confirmed by simulations and represents a key factor for understanding the evolutionary dynamics in heterogeneous populations.

Based on our approach, Eqs. (7) and (8) allow one to easily determine and compare fixation probabilities on general graphs for different games. In order to compare our results with previous studies [5,7], let us turn to the prisoner dilemma, which is widely employed in studying evolution of cooperation. In every interaction, cooperators, $C$, pay a cost $c$ and provide a benefit $b$ to their partner $(b>c)$. Defectors, $D$, pay no cost and provide no benefits. The payoff matrix becomes

$$
\begin{gathered}
C \\
C \\
D
\end{gathered}\left(\begin{array}{cc}
b-c & -c \\
b & 0
\end{array}\right) .
$$

Figure 2 shows the results of fixation probability, $\rho_{C}$, on regular random graphs and scale-free networks. The average degree is $k=4$, and we use two different cost-to-benefit ratios; in line with the $b / c>k$ rule [5], for $b / c=10$ natural selection favors cooperation but favors defectors for $b / c=2$. Comparisons with simulation results reveal good predictions for both types of graphs but predictions are better and extend to higher selection strengths on regular random graphs than on scale-free networks. With increasing selection strength, the coupling between the game dynamics and the underlying graph topologies becomes stronger on scale-free networks than on regular random graphs; thus the effect of degree heterogeneity on the tolerance of our approach comes much more prominent, since here we simply use empirically determined correlations under neutral evolution. In particular, our approach returns better predictions than pair approximation (Fig. 2), while inclusive fitness analysis cannot directly predict the probability of fixation. Thus, our approach provides not only a powerful numerical technique but also a convenient framework for studying games on graphs.

The critical $b / c$ ratio, above which cooperation is favored by natural selection, i.e., $\rho_{C}>1 / N$, is shown in Fig. 3. For weak selection, $\rho_{A}>1 / N$ is equivalent to $\rho_{A}>\rho_{B}$ provided that the payoff matrix satisfies the condition equal gains from switching (i.e., $M_{A A}+M_{B B}=M_{A B}+M_{B A}$ [33]) [7] and holds for the payoff matrix (9). Our results are in good agreement with exact numerical simulations. In particular, they exceed the average graph degree $k$, reflecting the finite-size effects (Fig. 3). For regular random graphs, the results fit well with theoretical predictions [7] [Fig. 3(a)] and are in agreement with pair approximation in the limit of large populations and weak selection (see Appendix A). Besides, we find degreeheterogeneity results in higher critical $b / c$ ratios for cooperation to be favored on scale-free networks, as compared to regular random graphs [Fig. 3(b)]. This result can be understood as follows: selection always works against the initial increase in cooperators but degree heterogeneity additionally opposes the spreading of rare types as demonstrated in Fig. 1(c). More specifically, consider a star graph as an illustrative example. A star graph is an extreme form of a heterogeneous graph, where all periphery nodes are connected to a single central hub. Both simulations and analytical derivations show that the fixation probability $\rho_{C}$ of a randomly placed cooperator is lower than $1 / N$ for any $b / c$ ratio and any nonzero intensity of selection (see Appendix B). This suggests that heterogeneous graphs generally impose higher invasion barriers than regular graphs, which results in a higher critical $b / c$ ratio to compensate for the unfavorable 

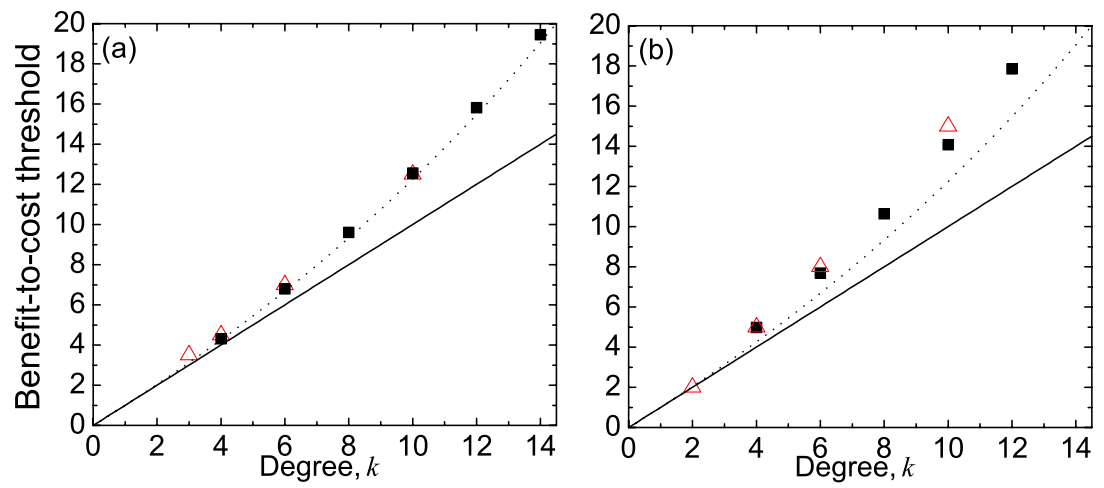

FIG. 3. (Color online) Evolution of cooperation in the prisoner dilemma; the benefit-to-cost, $b / c$, threshold ( $\mathbf{\square}$ : our approach; $\triangle$ : simulations), above which cooperators have a selective advantage over defectors, versus degree $k$ on (a) random regular graphs and (b) scale-free networks. Analytical prediction with finite-size correction (dotted line) [7] [i.e., $b / c=k(N-2) /(N-2 k)]$ and prediction from pair approximation (solid line) [5] (i.e., $b / c=k) .(N=100$ and $w=0.01$.)

initial conditions. Noteworthy, it is reported that a heterogeneous graph is an inhospitable environment for a mutant to evolve in the case of constant selection [21]. Complementing this conclusion, our results show that it remains true for weak selection. Paradoxically, previous investigations revealed that scale-free networks provide a hospitable environment for cooperation $[4,19]$; however, this conclusion hinges on the simultaneous appearance of a number of cooperators to overcome the invasion barrier. In addition, the high cooperation level is attributed to the dynamical organization of cooperators and defectors, which strongly depends on the network topology and the game parameters [19]. Otherwise, one would expect that invasion attempts of cooperators are doomed under strong selection. Actually, for the updating rule used in Ref. [4], cooperation never evolves from a single cooperator.

Thus far, we have demonstrated that degree heterogeneity generally impedes the fixation of a single mutant cooperator in the prisoner dilemma under weak selection. For a more comprehensive insight let us consider the (quasi) stationary frequency of cooperators on scale-free networks in the two limiting cases of weak selection versus strong selection. In both cases, we use an individual accumulated payoff accrued from interactions with all its neighbors. To ensure non- negative fitness values, we rescale the payoff matrix (9) as $M_{A A}=1, M_{A B}=0, M_{B A}=1+c / b$, and $M_{B B}=c / b(c / b$ denotes the cost-to-benefit ratio). Note that for strong selection very different results are expected when using different payoff accounting mechanisms (e.g., accumulated or normalized payoff [39]) in heterogeneous graphs. In order to consider stationary distributions, we introduce a small mutation rate, $\mu$, such that the system can escape the absorbing states (all $C$ and all $D$ ). With probability $1-\mu$ the offspring keeps the strategy of the parent but with probability $\mu$ and the offspring adopts the opposite strategic type. As mentioned before, if $A$ has a selective advantage over $B$, that is, $\rho_{A}>\rho_{B}$ in terms of fixation probabilities, $A$ will be more abundant than $B$ in the long run for rare mutants. In other words, the stationary frequency of $A$ should be larger than 0.5 if $A$ is favored over $B$ in this mutation-selection process.

Figures 4(a) and 4(b) show the stationary distribution of states in scale-free networks for weak and strong selections, respectively. The cost-to-benefit ratio $b / c=2$ is less than the average graph degree 4, and hence weak selection favors defection [5]. Consequently, the stationary frequency of cooperators $(0.495)$ lies slightly below 0.5 . For strong selection, cooperation is further inhibited and the stationary frequency of cooperators is about 0.123 . The apparent sym-
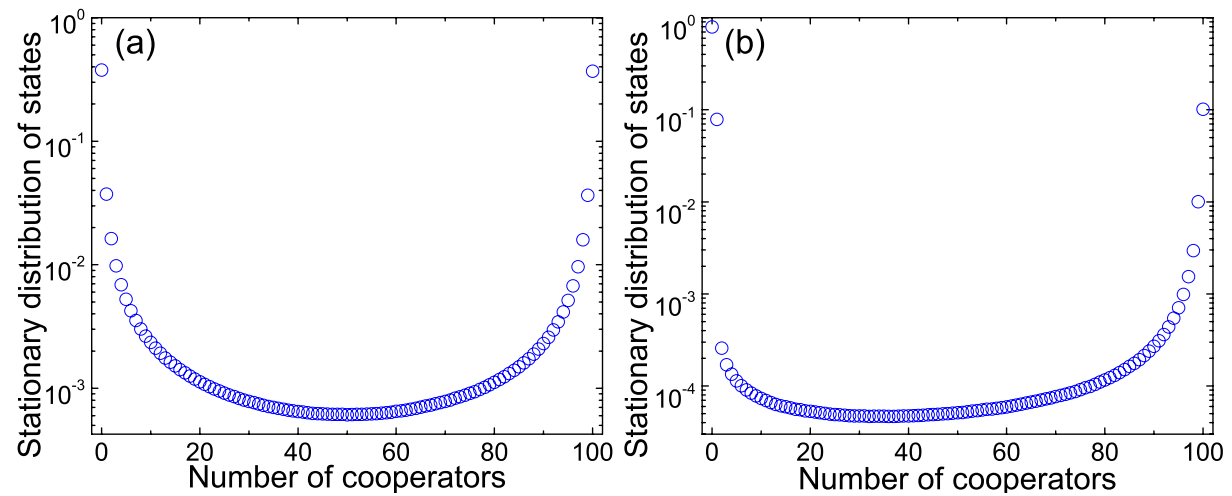

FIG. 4. (Color online) Stationary distribution of the mutation-selection process for (a) weak selection $w=0.001$ and for (b) strong selection $w=0.999$ on scale-free networks. Panels (a) and (b) show stationary distribution of states for the prisoner dilemma on scale-free networks with $b / c=2$. The stationary frequency of cooperators is (a) 0.495 and (b) 0.123 . See text for details. (Mutation rate $\mu=10^{-3}, N$ $=100$, and $k=4$. The results are averaged over $10^{5}$ independent runs, each sampling time $T=10^{4}$.) 
metric distribution of states under weak selection is strongly skewed by increasing selection strength [cf. Figs. 4(a) and 4(b)]. Interestingly, for strong selection, this result indicates that once rare cooperators successfully overcome the invasion barrier imposed by the degree heterogeneity and the increasing selection pressure, the system is more likely to consist of more abundant cooperators than defectors. Nevertheless, strong selection makes it even harder for rare cooperators to get over this invasion barrier. As a consequence, the system spends much more time in the all- $D$ state than in the all- $C$ state. Most interestingly, if we average over intermediate numbers of cooperators, say from 10 to 90 , the fraction of cooperators (0.596) is enhanced under strong selection, as compared to weak selection (0.499). In fact, previous studies $[4,19]$ start from half-to-half mixing of cooperators and defectors, and measure the dynamic equilibrium (quasistationary) frequency of cooperators as an indicator of whether or not cooperation is promoted on various types of networks under strong selection. In these studies, the large population size and the type of games result in exceedingly long fixation times that render a mutation-selection framework unfeasible. Our present work complements existing studies [4,18,19,21] and furthers our understanding of the evolutionary dynamics on heterogeneous graphs.

To illustrate the power of our numerical techniques we demonstrate the influence of the network topology on evolutionary dynamics for general payoff matrices. Following previous studies [40], we normalize the payoff matrix (1) such that $M_{A A}=1, M_{A B}=S, M_{B A}=T$, and $M_{B B}=0(-1 \leq S \leq 1$ and $0 \leq T \leq 2)$. The type of game is determined by the region in the $(S, T)$ plane. For $T>1$ and $S<0$, this is the prisoner dilemma; for $T>1$ and $S>0$, this is the snowdrift game; for $T<1$ and $S<0$, this is the stag-hunt game; for $T<1$ and $S$ $>0$, this is the (by-product) mutualism. Thus, this payoff matrix can represent different social dilemmas [41]. The ratio of the fixation probability of $A$ and of $B, \rho_{A} / \rho_{B}$, is shown as a function of $S, T$ for random regular graphs, random graphs [37], and scale-free networks [38], respectively (Fig. 5). Three types of graphs exhibit an increasing amount of degree heterogeneity. In each case, we are interested in the critical values of $T$ and $S$ such that $\rho_{A}=\rho_{B}$. For smaller $T$ or larger $S$, we have $\rho_{A}>\rho_{B}$; that is, $A$ is more abundant than $B$ for rare mutants in the stationary distribution of mutation-selection dynamics.

In principle, it is rather time consuming to determine these critical values of $S, T$ as this requires the fixation probability of $A$ and $B$, respectively. Furthermore, the unconditional fixation time depends crucially on the type of game under study [31]. In sharp contrast, our approach is quite efficient; we only need to simulate neutral evolution for each type of graph and then derive the evolutionary outcome for every point in the $(S, T)$ plane. For all three types of network structures we find excellent agreement between our predictions [dashed lines in Figs. 5(b)-5(d)] and the actual simulation results [circles in Figs. 5(b)-5(d)]. Note that the regime with $\rho_{A}>\rho_{B}$ is largest on random regular graphs and decreases with increasing heterogeneity, i.e., it is smaller on random graphs and still smaller on scale-free networks. The critical $T$ as a function of $S$ appears to follow a straight line of slope 1 in the $(S, T)$ plane. The position of this line reflects the influence of specific population structures on the evolutionary dynamics. For star graphs this line passes through $(0,1)$, which means that star graphs provide less hospitable environments for cooperation than scale-free networks. As a reference, Fig. 5(a) depicts results for well-mixed populations where the critical line for $\rho_{A}=\rho_{B}$ under weak selection passes through $\left(0, \frac{N-2}{N}\right)$ ( $N$ is the population size; see Ref. [24] for a detailed analysis). Overall, these results demonstrate that while spatial structures generally promote the evolution of cooperation, degree heterogeneity generally opposes cooperation in social dilemmas.

Finally, analyzing the evolutionary dynamics of other games does not require further simulations. Let $A$ and $B$ denote the strategies tit for tat (in which the player cooperates in the first round and then copies whatever the opponent did in the previous round) and "always to defect" (AllD), respectively, in an iterated prisoner dilemma with $n$ rounds on average. The payoff matrix is $M_{A A}=n(b-c), M_{A B}=-c$, $M_{B A}=b$, and $M_{B B}=0$. Let $b / c=1.5$, such that for $n>3$, this is a coordination (bistable) game between tit for tat and AllD. Note that rescaling the payoffs as $R=M_{A A}=1, S$ $=M_{A B}=-c /[n(b-c)], T=M_{B A}=b /[n(b-c)]$, and $P=M_{B B}=0$ maps this coordination game to the Stag-Hunt quadrant in the $(S, T)$ plane shown in Fig. 5. In this case $T+S=1 / n$ holds, and thus for fixed $n$ games between tit for tat and AllD are represented by a line that is perpendicular to the critical line for $\rho_{A}=\rho_{B}$ in the $(S, T)$ plane. The intersection of these two lines marks the critical $b / c$ ratio for selection to favor tit for tat replacing AllD. In well-mixed populations, this occurs if $M_{A A}+2 M_{A B}>M_{B A}+2 M_{B B}$ [24]. This condition, coined as "1/3 rule" in Ref. [24], corresponds to strategy $A$ having larger fitness than $B$ at a frequency $1 / 3$. We should point out that this $1 / 3$ rule corresponds to the dashed line plotted in Fig. 5(a); here this requires $n>7$ as explicitly shown in Fig. 6. On regular random graphs and for fixed $b / c$ ratio, there exists a critical minimum number of rounds that allows selection of tit for tat (Fig. 6). In the limit of weak selection, the critical number of rounds monotonically increases with the degree $k$, gradually recovering the $1 / 3$ rule of well-mixed populations (i.e., fully connected graphs) (Fig. 6). Interestingly, depending on the number of rounds, the graph degree affects the evolution of tit for tat differently; for small $n$, increasing the number of interaction partners reduces the fixation probability of a single tit-for-tat player; in contrast, for large $n$, an increasing of number of interaction partners makes it easier for tit-for-tat players reaching fixation. The advantages of the tit-for-tat strategy become much more pronounced once tit-for-tat players have successfully established a cluster. In this case, tit-for-tat players greatly benefit from repeated interactions in a dense neighborhood (i.e., large degree). For small $n$, the benefit of assortment is not sufficient to offset the exploitation by defectors along the periphery. This effect is enhanced by increasing the number of neighbors. Conversely, sufficiently large $n$ stabilizes cooperation, and this positive effect is, to a large extent, further enhanced by increasing the neighborhood size. The nontrivial trade off between number of neighbors and number of rounds indicates an interesting interplay between game dynamics and graph topologies.

In summary, neutral evolution on arbitrary graphs returns the correlations between neighboring individuals and repre- 

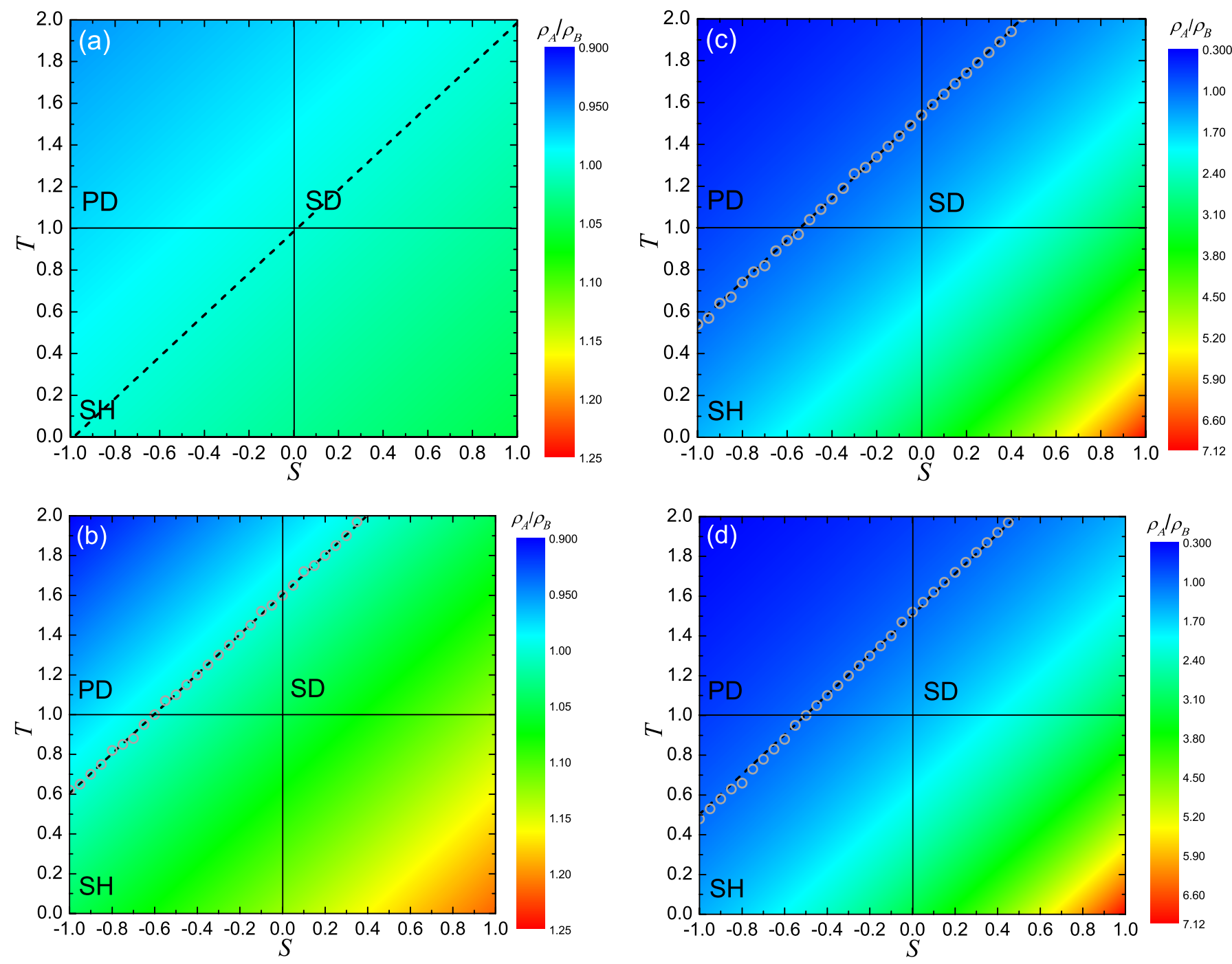

FIG. 5. (Color online) The comparison of fixation probabilities on different types of graphs. The payoff matrix under study is $M_{A A}=1$, $M_{A B}=S, M_{B A}=T$, and $M_{B B}=0(-1 \leq S \leq 1$ and $0 \leq T \leq 2)$. For $T>1$ and $S<0$, this is the prisoner dilemma (PD); for $T>1$ and $S>0$, this is the snowdrift game (SD); for $T<1$ and $S<0$, this is the stag-hunt game (SH); for $T<1$ and $S>0$, this is the (by-product) mutualism. As a reference, panel (a) shows the comparison of fixation probabilities in well-mixed populations. The type of graph under consideration is (b) random regular graphs, (c) random graphs, and (d) scale-free networks. The ratio of fixation probability of $A$ to $B, \rho_{A} / \rho_{B}$, is shown as a contour in the two-dimensional (2D) parameter space $(S, T)$ using our approach. Note that the range of $\rho_{A} / \rho_{B}$ values increases with space and with increasing degree heterogeneity. The dashed line corresponds to the situation of $\rho_{A}=\rho_{B}$ predicted by our method. The circles $(O)$ represent the simulation results regarding the critical $T$ for each $S$ such that $\rho_{A}=\rho_{B}$. In our simulations, the fixation probability of $A(B)$ is determined by the fraction of runs where $A(B)$ reached fixation out of $10^{7}$ runs. (Population size $N=100$ and average degree $k=4$. Selection pressure $w=5 \times 10^{-3}$.)

sents the backbone of our approach to model evolutionary dynamics in the limit of weak selection based on microscopic descriptions of the transitions along the interface of the two competing types. In particular, degree heterogeneity generally increases the invasion barrier for rare mutants. Consequently, for a mutant cooperator to thrive in a world of defectors, regular graphs provide a more hospitable environment than scale-free networks, for example. Furthermore, the emergence of the conditional cooperative strategy tit for tat strongly depends on the tradeoff between the graph degree and the number of rounds.

\section{ACKNOWLEDGMENTS}

We are grateful for helpful comments from two anonymous referees. We thank $\mathrm{H}$. Ohtsuki for comments on an earlier version of this paper. We acknowledge financial support from NSFC under Grant Nos. 60674050, 60736022, and 60528007 (F.F. and L.W.), the John Templeton Foundation, the NSF/NIH joint program in mathematical biology (C.H. and M.A.N.), and NSERC (C.H.). The Program for Evolutionary Dynamics at Harvard University is sponsored by $\mathrm{J}$. Epstein. 


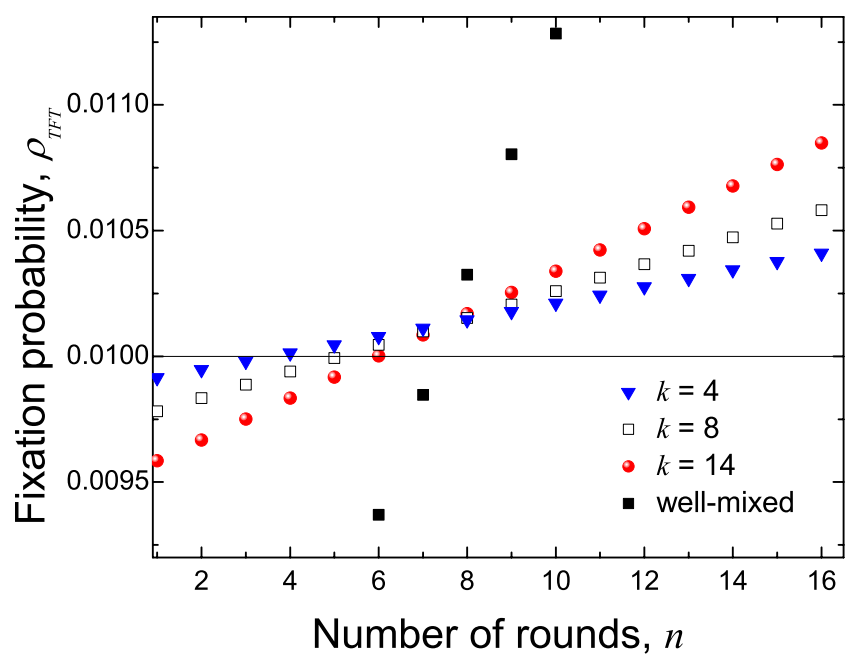

FIG. 6. (Color online) Tit for tat (TFT) vs AllD in the iterated prisoner dilemma. The fixation probability of a tit-for-tat mutant as a function of number of rounds, $n$, on random regular graphs with $k=4(\boldsymbol{\nabla}), k=8(\square), k=14(\bullet)$, as well as in well-mixed populations (ם). Depending on the degree $k$, there exists a critical minimum number of rounds, $n$, such that selection favors tit for tat. $(N=100$, $b / c=1.5$, and $w=10^{-4}$.)

\section{APPENDIX A: USING PAIR APPROXIMATION IN OUR APPROACH}

Let us consider evolutionary games on regular graphs with degree $k$. Here we show that our present approach recovers the results obtained in Ref. [5] with pair approximation in the limit of large populations and weak selection. To do this, we adopt analytical pair-approximated local configurations in neutral case instead of simulation-determined data. Note that pair approximation is exact for infinite degreeregular graphs without loops (e.g., Cayley trees) and cannot correct finite-size effects.

Let us denote by $p_{A}=i / N\left(p_{B}\right)$ the abundance of $A(B)$. Let $p_{X Y}$ represent the probability of finding an $X Y$ pair in the system, and let $q_{Y \mid X}$ be the conditional probability to find a $Y$ player given that the adjacent node is occupied by an $X$ player. Here, both $X$ and $Y$ stand for either $A$ or $B$. We find the same local configurations for death-birth and birth-death updatings under neutral evolution. Given an initial frequency of $A, p_{A}(0)$, the expected change in $p_{A}(t)$ is equal to 0 at all time $t$, i.e., $p_{A}(t) \equiv p_{A}(0)$. Furthermore, after some algebra (detailed derivations can be found in Ref. [5]) we can obtain the equilibrium $p_{X Y}$ and $q_{Y \mid X}$ as follows, respectively:

$$
\begin{gathered}
p_{X Y}=\frac{1}{k-1} \frac{p_{X}+p_{Y}}{2} \delta_{X, Y}+\frac{k-2}{k-1} p_{X} p_{Y} \\
q_{Y \mid X}=\frac{1}{k-1} \delta_{X, Y}+\frac{k-2}{k-1} p_{Y} .
\end{gathered}
$$

Note that under neutral evolution equilibrium $p_{X Y}$ and $q_{Y \mid X}$ are only dependent of $p_{A}(0)$ and the degree $k$.

Let us now couple the game in by calculating individual payoffs according to these equilibrium local configurations. That is,

$$
\Pi_{X}^{Y}=\left[\delta_{X, A}+(k-1) q_{A \mid Y}\right] M_{Y A}+\left[\delta_{X, B}+(k-1) q_{B \mid Y}\right] M_{Y B},
$$

where $\Pi_{X}^{Y}$ denotes the payoff of a $Y$ player within the focal $X$-player neighborhood. The corresponding fitness is $F_{X}^{Y}=1$ $-w+w \Pi_{X}^{Y}$.

For death-birth updating, the transition matrix for the number of $A$ individuals $i$ in this process is given by

$$
\begin{aligned}
& T_{A}^{+}(i)=p_{B} \frac{q_{A \mid B} F_{B}^{A}}{q_{A \mid B} F_{B}^{A}+q_{B \mid B} F_{B}^{B}}, \\
& T_{A}(i)=p_{A} \frac{q_{B \mid A} F_{A}^{B}}{q_{A \mid A} F_{A}^{A}+q_{B \mid A} F_{A}^{B}} .
\end{aligned}
$$

Moreover the number of $A$ individuals remains constant with probability $T_{A}^{0}(i)=1-T_{A}^{+}(i)-T_{A}^{-}(i)$.

In parallel, for birth-death updating, the transition probabilities become

$$
\begin{aligned}
& T_{A}^{+}(i)=\frac{p_{A} F_{B}^{A}}{\sum_{j=1}^{N} F_{j}} q_{B \mid A}, \\
& T_{A}(i)=\frac{p_{B} F_{A}^{B}}{\sum_{j=1}^{N} F_{j}} q_{A \mid B},
\end{aligned}
$$

where $\sum_{j=1}^{N} F_{j}$ represents the total fitness of the whole population.

In the limit of weak selection, using Taylor-series expansion at $w=0$ and neglecting the term $O\left(w^{n}\right)(n \geq 2)$, we obtain the following conditions for death-birth and birth-death update mechanisms, respectively:

$$
\rho_{A}>1 / N \Leftrightarrow \begin{cases}\text { death-birth: } & \sum_{j=1}^{N-1} \sum_{i=1}^{j} q_{B \mid B}\left(\Pi_{B}^{A}-\Pi_{B}^{B}\right)+q_{A \mid A}\left(\Pi_{A}^{A}-\Pi_{A}^{B}\right)>0 \\ \text { birth-death: } & \sum_{j=1}^{N-1} \sum_{i=1}^{j}\left(\Pi_{B}^{A}-\Pi_{A}^{B}\right)>0,\end{cases}
$$




$$
\frac{\rho_{A}}{\rho_{B}}>1 \Leftrightarrow \begin{cases}\text { death-birth: } & \sum_{i=1}^{N-1} q_{B \mid B}\left(\Pi_{B}^{A}-\Pi_{B}^{B}\right)+q_{A \mid A}\left(\Pi_{A}^{A}-\Pi_{A}^{B}\right)>0 \\ \text { birth-death: } & \sum_{i=1}^{N-1}\left(\Pi_{B}^{A}-\Pi_{A}^{B}\right)>0 .\end{cases}
$$

Introducing the notation $x=j / N$ and $p_{A}=i / N$, we find in the continuum limit that the sums in inequalities (A8) and (A9), $\Sigma_{j=1}^{N-1} \Sigma_{i=1}^{j}(\cdot)$ and $\Sigma_{i=1}^{N-1}(\cdot)$, can be estimated as $\int_{0}^{1} d x \int_{0}^{x} d p_{A}(\cdot)$ and $\int_{0}^{1} d p_{A}(\cdot)$, respectively. Using Eqs. (A1) and (A2) we obtain

$$
\begin{aligned}
& \rho_{A}>1 / N \Leftrightarrow \begin{cases}\text { death-birth: } & (k+1)^{2} M_{A A}+\left(2 k^{2}-2 k-1\right) M_{A B}-\left(k^{2}-k+1\right) M_{B A}-\left(2 k^{2}+k-1\right) M_{B B}>0 \\
\text { birth-death: } & (k+1) M_{A A}+(2 k-1) M_{A B}-(k+1) M_{B A}-(2 k-1) M_{B B}>0,\end{cases} \\
& \frac{\rho_{A}}{\rho_{B}}>1 \Leftrightarrow \begin{cases}\text { death-birth: } & (k+1) M_{A A}+(k-1) M_{A B}-(k-1) M_{B A}-(k+1) M_{B B}>0 \\
\text { birth-death: } & M_{A A}+M_{A B}-M_{B A}-M_{B B}>0 .\end{cases}
\end{aligned}
$$

Hereby, using discrete Markov chain we obtain the same results derived from diffusion approximation in Ref. [5] for large populations and weak selection.

\section{APPENDIX B: FIXATION PROBABILITIES ON STAR GRAPHS}

We first consider neutral evolution on star graphs. For the simplicity of notation, consider a star graph of $N+1$ nodes with a center node and $N$ periphery nodes. Let $P_{i}^{1}\left(P_{i}^{0}\right)$ be the probability of fixation of $A$ given there are $i A$ 's in the leaves and an $A$ in the center (a $B$ in the center). Here, we consider death-birth update mechanism only. Hence, the fixation probabilities can be solved by following two recurrence equations:

$$
\begin{aligned}
P_{i}^{1}= & \frac{N-i}{N+1} P_{i+1}^{1}+\frac{1}{N+1} \frac{N-i}{N} P_{i}^{0} \\
& +\left(1-\frac{N-i}{N+1}-\frac{1}{N+1} \frac{N-i}{N}\right) P_{i}^{1},
\end{aligned}
$$

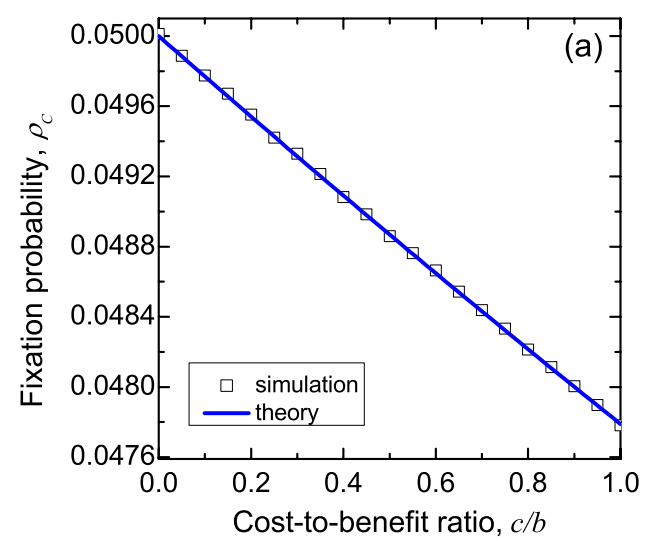

$$
P_{i}^{0}=\frac{i}{N+1} P_{i-1}^{0}+\frac{1}{N+1} \frac{i}{N} P_{i}^{1}+\left(1-\frac{i}{N+1}-\frac{1}{N+1} \frac{i}{N}\right) P_{i}^{0},
$$

with boundary conditions $P_{0}^{0}=1$ and $P_{N}^{1}=1$.

We have

$$
\left[\begin{array}{c}
P_{i}^{1} \\
P_{i}^{0}
\end{array}\right]=\left[\begin{array}{cc}
\frac{i+N}{N} & -\frac{i}{N} \\
\frac{i}{N} & \frac{-i+N}{N}
\end{array}\right]\left[\begin{array}{l}
P_{0}^{1} \\
P_{1}^{0}
\end{array}\right]
$$

Thus, we obtain $P_{0}^{1}=\frac{1}{2}$ and $P_{1}^{0}=\frac{1}{2 N}$.

Now we turn to the frequency-dependent fitness in deathbirth update. Consider a symmetric $2 \times 2$ game between $A$ and $B$ according to matrix (1). Let $f_{A}$ and $f_{B}\left(g_{A}\right.$ and $\left.g_{B}\right)$ be the fitness of $A$ and $B$ given that the hub is occupied by an $A(B)$. We have

$$
f_{A}=1-w+w \cdot M_{A A}
$$

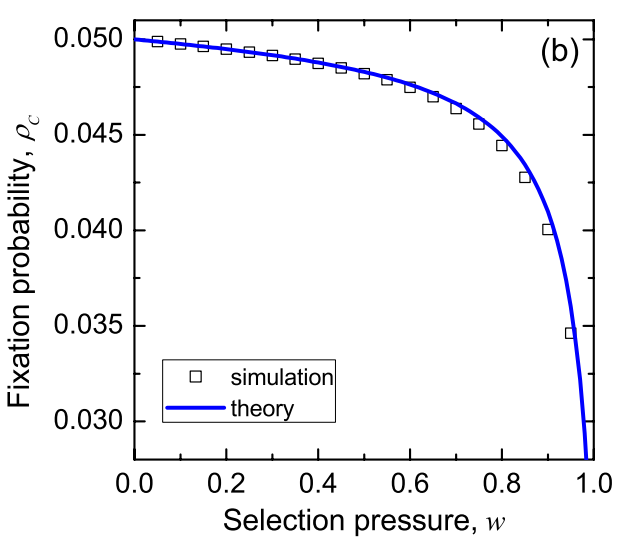

FIG. 7. (Color online) Fixation probability of a randomly placed cooperator, $\rho_{C}$, in the prisoner dilemma on star graphs. (a) $\rho_{C}$ as a function of cost-to-benefit ratio $c / b$ for fixed intensity of selection $w=0.05$; (b) $\rho_{C}$ as a function of selection pressure $w$ for fixed $c / b$ $=0.05$. Empty squares represent simulation data and solid lines represent theoretical results. Population size is 20 , and the fixation probability from simulations is determined by the fraction of runs where cooperators reached fixation out of $10^{8}$ runs. 


$$
\begin{aligned}
& f_{B}=1-w+w \cdot M_{B A}, \\
& g_{A}=1-w+w \cdot M_{A B}, \\
& g_{B}=1-w+w \cdot M_{B B},
\end{aligned}
$$

where $w$ represents the intensity of selection, $w \in[0,1]$. Then the Markov process can be expressed as

$$
\begin{aligned}
P_{i}^{1}= & \frac{N-i}{N+1} P_{i+1}^{1}+\frac{1}{N+1} \frac{(N-i) f_{B}}{(N-i) f_{B}+i f_{A}} P_{i}^{0} \\
& +\left(1-\frac{N-i}{N+1}-\frac{1}{N+1} \frac{(N-i) f_{B}}{(N-i) f_{B}+i f_{A}}\right) P_{i}^{1},
\end{aligned}
$$

$$
\begin{aligned}
P_{i}^{0}= & \frac{i}{N+1} P_{i-1}^{0}+\frac{1}{N+1} \frac{i g_{A}}{(N-i) g_{B}+i g_{A}} P_{i}^{1} \\
& +\left(1-\frac{i}{N+1}-\frac{1}{N+1} \frac{i g_{A}}{(N-i) g_{B}+i g_{A}}\right) P_{i}^{0},
\end{aligned}
$$

with boundary conditions $P_{0}^{0}=0$ and $P_{N}^{1}=1$. Accordingly, $P_{0}^{1}$ and $P_{1}^{0}$ can be analytically determined by solving the above two coupled recurrence equations. Both simulations and analytical results show that the fixation probability $\rho_{C}$ of a randomly placed cooperator is lower than neutral case for any $b / c$ ratio and any nonzero intensity of selection (Fig. 7).
[1] G. Abramson and M. Kuperman, Phys. Rev. E 63, 030901(R) (2001).

[2] H. Ebel and S. Bornholdt, Phys. Rev. E 66, 056118 (2002).

[3] B. J. Kim, A. Trusina, P. Holme, P. Minnhagen, J. S. Chung, and M. Y. Choi, Phys. Rev. E 66, 021907 (2002).

[4] F. C. Santos and J. M. Pacheco, Phys. Rev. Lett. 95, 098104 (2005).

[5] H. Ohtsuki, C. Hauert, E. Lieberman, and M. A. Nowak, Nature (London) 441, 502 (2006).

[6] H. Ohtsuki, M. A. Nowak, and J. M. Pacheco, Phys. Rev. Lett. 98, 108106 (2007).

[7] P. D. Taylor, T. Day, and G. Wild, Nature (London) 447, 469 (2007).

[8] M. A. Nowak, Evolutionary Dynamics: Exploring the Equations of Life (Harvard University, Cambridge, MA, 2006).

[9] M. A. Nowak and R. M. May, Nature (London) 359, 826 (1992).

[10] C. Hauert and M. Doebeli, Nature (London) 428, 643 (2004).

[11] M. Nakamaru, H. Matsuda, and Y. Iwasa, J. Theor. Biol. 184, 65 (1997).

[12] G. Szabó and C. Tőke, Phys. Rev. E 58, 69 (1998).

[13] G. Szabó, J. Vukov, and A. Szolnoki, Phys. Rev. E 72, 047107 (2005).

[14] C. Hauert and G. Szabó, Am. J. Phys. 73, 405 (2005).

[15] G. Szabó and C. Hauert, Phys. Rev. Lett. 89, 118101 (2002).

[16] T. Reichenbach, M. Mobilia, and E. Frey, Nature (London) 448, 1046 (2007).

[17] F. C. Santos, M. D. Santos, and J. M. Pacheco, Nature (London) 454, 213 (2008).

[18] F. C. Santos, J. M. Pacheco, and T. Lenaerts, Proc. Natl. Acad. Sci. U.S.A. 103, 3490 (2006).

[19] J. Gómez-Gardeñes, M. Campillo, L. M. Floría, and Y. Moreno, Phys. Rev. Lett. 98, 108103 (2007).

[20] J. M. Pacheco, A. Traulsen, and M. A. Nowak, Phys. Rev. Lett. 97, 258103 (2006).
[21] T. Antal, S. Redner, and V. Sood, Phys. Rev. Lett. 96, 188104 (2006).

[22] E. Lieberman, C. Hauert, and M. A. Nowak, Nature (London) 433, 312 (2005).

[23] G. Szabó and G. Fáth, Phys. Rep. 446, 97 (2007).

[24] M. A. Nowak, A. Sasaki, C. Taylor, and D. Fudenberg, Nature (London) 428, 646 (2004).

[25] C. P. Roca, J. A. Cuesta, and A. Sánchez, Phys. Rev. Lett. 97, 158701 (2006).

[26] A. Traulsen, J. C. Claussen, and C. Hauert, Phys. Rev. Lett. 95, 238701 (2005).

[27] J. C. Claussen and A. Traulsen, Phys. Rev. Lett. 100, 058104 (2008).

[28] M. Kimura, The Neutral Theory of Molecular Evolution (Cambridge University Press, Cambridge, England, 1983).

[29] G. Wild and A. Traulsen, J. Theor. Biol. 247, 382 (2007).

[30] P. A. P. Moran, The Statistical Processes of Evolutionary Theory (Clarendon, Oxford, 1962).

[31] A. Traulsen, J. M. Pacheco, and M. A. Nowak, J. Theor. Biol. 246, 522 (2007).

[32] H. Matsuda, N. Ogita, A. Sasaki, and K. Satō, Prog. Theor. Phys. 88, 1035 (1992).

[33] M. A. Nowak and K. Sigmund, Acta Appl. Math. 20, 247 (1990).

[34] L. E. Blume, Games Econ. Behav. 5, 387 (1993).

[35] J. Miekisz, Lect. Notes Math. 1940, 269 (2008).

[36] S. Karlin and H. M. A. Taylor, A First Course in Stochastic Processes, 2nd ed. (Academic, London, 1975).

[37] B. Bollobás, Random Graphs (Academic, New York, 1995).

[38] A.-L. Barabási and R. Albert, Science 286, 509 (1999).

[39] A. Szolnoki, M. Perc, and Z. Danku, Physica A 387, 2075 (2008).

[40] C. Hauert, Proc. R. Soc. London, Ser. B 268, 761 (2001).

[41] C. Hauert, F. Michor, M. A. Nowak, and M. Doebeli, J. Theor. Biol. 239, 195 (2006). 